\title{
Changes in calcitonin gene-related peptide expression following joint immobilization in rats
}

Tomohiko Nishigami $^{\mathrm{a}}$, Yoji Osako ${ }^{\mathrm{b}}$, Kenjiro Tanaka ${ }^{\mathrm{b}}$ Kazunari Yuri ${ }^{\mathrm{b}}$, Motohiro Kawasaki ${ }^{\mathrm{c}}$, Tatsunori

Ikemoto $^{\mathrm{c}}$, Matthew McLaughlin ${ }^{\mathrm{d}}$, Kenji Ishida $^{\mathrm{a}}$, Toshikazu Tani ${ }^{\mathrm{c}}$, Takahiro Ushida ${ }^{\mathrm{e}}$ *

a Rehabilitation Center, Kochi Medical School Hospital, Nankoku, Japan

b Department of Neurobiology \&Anatomy, Kochi Medical School, Nankoku, Japan

c Department of Orthopaedic Surgery, Kochi Medical School, Nankoku, Japan

d Nankoku Pain Research Group, Kochi Medical School, Kochi, Japan

e Multidisciplinary Pain Center, Aichi Medical University, Nagakute, Japan

*Corresponding author. Multidisciplinary Pain Center, Aichi Medical University, Nagakute, Aichi

480-1195, Japan. Tel \& Fax: +81 56162 5004. E-mail address: ushidat-koc@umin.ac.jp (T. Ushida).

Number of text pages (including figures and tables); 17

Number of figures; 2

Number of tables; 0 
Long-term immobilization by casting can occasionally cause pathologic pain states in the immobilized side. The underlying neurophysiological mechanisms of immobilization-related pain are not well understood. For this reason, we specifically examined changes of calcitonin gene-related peptide (CGRP) expression in the dorsal root ganglion (DRG), spinal dorsal horn and posterior nuclei (cuneate nuclei) in a long-term immobilization model following casting for 5 weeks. A plastic cast was wrapped around the right limb from the forearm to the forepaw to keep wrist joint at $90^{\circ}$ of flexion. In this model, CGRP in immobilized (ipsilateral) side was distributed in larger DRG neurons compared with contralateral side, even though the number of CGRP-immunoreactive (CGRP-IR) neurons did not differ. Spinal laminae III-V, not laminae I-II in ipsilateral side showed significantly high CGRP expression relative to contralateral side. CGRP expression in cuneate nuclei was not significantly different between ipsilateral and contralateral sides. Long-term immobilization by casting may induce phenotypic changes in CGRP expression both in DRG and spinal deep layers, and these changes are partly responsible for pathological pain states in immobilized side.

Keywords: long-term joint immobilization, pain, calcitonin gene-related peptide, dorsal root ganglion, spinal dorsal horn, posterior nuclei 
Casting (immobilization) is commonly utilized because it is considered to be useful in restoring injured organs by local resting. However, long-term immobilization results in harmful complications such as muscular atrophy or joint contracture in the immobilized side. In addition, recent research reveals that immobilization may cause not only motor dysfunction but also sensory-motor or autonomic nerve dysfunction, and occasionally immobilization-related pain $[5,19,26]$. With regard to immobilization-related neurological dysfunction, Butler and colleagues (1999) reported observing signs of neglect-like states and complex regional pain syndromes after casting limbs of patients with foot fractures and healthy volunteers [2]. Therefore, long-term immobilization may limit therapeutic outcome and may produce low levels of activity of daily living.

Predominantly, immobilization-related pain has been considered to be a result of shortened muscle and the adhesion of joints and periarticular structures. Clinically, surgical release of the periarticular tissues has been successful in helping relieve patients with painful post-traumatic contracture [3]. Previously, we developed a long-term joint immobilization model, which showed a joint contracture, disuse tendencies, and pain behaviors in the affected limb, and we observed changes in electrophysiological responses of dorsal horn neurons to mechanical stimuli and motion stimuli [27]. This observation suggests that central changes as well as local peripheral changes are implicated in immobilization-related pain. It is well known that intense nociceptive stimulation (e.g., inflammation, nerve injury and cancer) alters the character of neurons in the central nervous system $[1,7,28]$ and these alterations are considered to be the 
cause of allodynia and hyperalgesia [22,24]. However, it remains unclear how these central neuronal changes occur under conditions of sensory input deprivation (long-term immobilization).

In the present study, we have focused on the expression of calcitonin gene-related peptide (CGRP).

CGRP has a wide distribution throughout the central and peripheral systems and is involved in processing nociceptive information. Firstly, in the spinal cord, CGRP is released from C and A $\delta$ nociceptive afferent fibers as an excitatory neurotransmitter and neuromodulator following painful stimuli, and sensitizes dorsal horn neurons $[6,20]$. In the experimental animals showing mechanical allodynia and hyperalgesia, CGRP expression in the spinal dorsal horn was markedly up-regulated $[9,15]$. Secondly, changes in CGRP expression within the dorsal root ganglion (DRG) have been studied in various pain models. The number of CGRP-immunoreactive (CGRP-IR) DRG neurons increased or decreased in inflammatory and neuropathic pain models $[11,14,21]$. In these studies, phenotypic changes in DRG CGRP expression have included both a change in the number of CGRP-IR neurons and a switch to CGRP expression in small or large DRG neurons. Interestingly, in neuropathic pain models, CGRP expression in the posterior nuclei (where information is conveyed by thick myelinated fibers encoding tactile sensation relayed to higher levels) significantly increased in the injured side, suggesting that CGRP released in non-nociceptive pathways is implicated in pain states [11,12]. The aim of the present study was to examine whether the expression of CGRP in DRGs, spinal dorsal horn, and posterior nuclei (cuneate nuclei) altered under conditions of long-term immobilization, to gain a better understanding of immobilization-related pain. 
All animal experiments were approved by the Kochi Medical School Animal Care and Use Committee and were performed at Kochi Medical School. Seven adult male Sprague-Dawley rats (250-300g; Japan SLC, Inc.) were used in this study. Long-term joint immobilization was maintained by using a plastic cast as described previously [27]. Briefly, the rats were anesthetized with tribromoethanol $200 \mathrm{mg} / \mathrm{kg}$ i.p.), and a plastic cast was wrapped around the right limb from the forearm to the forepaw to keep wrist joint at $90^{\circ}$ of flexion. After 5 weeks casting, the rats were perfused transcardially with $4 \%$ paraformaldehyde and DRGs, spinal cord, and medulla oblongata were excised at 24 hours after removing cast. Retrograde fluorogold labeling demonstrated that DRG neurons innervating the wrist joint in the rat were distributed throughout DRGs from C6 to Th1 levels, and most of the labeled neurons contained CGRP [8]. Hence, DRGs and spinal cords from C7 to Th1 levels were harvested and used for CGRP immunohistochemistry. All of the sections were cut on a cryostat at a thickness of $12 \mu \mathrm{m}$ (in DRG) and $23 \mu \mathrm{m}$ (in spinal cord and medulla oblongata), and were incubated in the primary antibody to CGRP (1:5000, Sigma) for 48 hours at $4^{\circ} \mathrm{C}$ and antibody labeling was detected using Alexa Fluor 488 (1:1000, Molecular Probes). For size distributions of the CGRP-IR neuronal profile, the areas of over 2000 DRG neurons were measured in each side, on both the immobilized (ipsilateral) and contralateral sides, using imaging analysis software (NIH Image). To calculate the number of CGRP-IR neurons, one hundred fluorescent images of ipsilateral and contralateral DRGs were extracted per animal at random (700 fluorescent images total each side), and the number of CGRP-IR neurons was counted. Only CGRP-IR neurons with visible nuclei 
were selected for measurement. The total area of CGRP fibers (pixels) in superficial layers (laminae I-II)

and deep layers (laminae III-V) in spinal dorsal horn and posterior nuclei (cuneate nuclei) in medulla

oblongata was quantitatively evaluated using NIH Image. The differences in the number of CGRP-IR

neurons and total area of CGRP fibers between the ipsilteral and contralateral sides were assessed using

unpaired t-tests. The differences in size distribution of DRG neurons were assessed using

Kolmogorov-Smirnov test. Statistically significant differences between groups were expressed as $p$ values

less than 0.05 .

No significant differences in the number of CGRP-IR DRG neurons were found between ipsilateral

(282.7 \pm 33.5$)$ and contralateral sides (279.5 \pm 33.7$)$. However, the size distribution of ipsilateral CGRP-IR

DRG neurons was significantly different from that of the contralateral side ( $<<0.0001$, Fig.1); ipsilateral

CGRP expression was observed in larger neurons on the contralateral side. The size distributions of

Nissl-stained DRG neurons were no different in the ipsilateral and contralateral sides $(\mathrm{p}=0.228)$,

demonstrating that all ipsilateral DRG neurons did not become larger, independent of CGRP expression.

In the spinal cord, the areas of ipsilateral CGRP-IR fibers significantly increased compared with the

contralateral side in laminae III-V (ipsilateral, $460028 \pm 89537$ pixels; contralateral, $365932 \pm 67834$ pixels:

$\mathrm{p}<0.05$ ) but not in laminae I-II (ipsilateral, $642202 \pm 78683$ pixels; contralateral, $620055 \pm 65095$ pixels: NS,

Fig.2). No significant change was observed in cuneate nuclei CGRP-IR fibers between the ipsilateral

( $33810 \pm 17762$ pixels) and contralateral sides (28683 \pm 13514 pixels). 
The present study revealed that long-term immobilization by casting induced an up-regulation of CGRP expression, not in superficial but in deep layers of dorsal horn and a shift in the distribution of CGRP expression profile to larger DRG neurons. When classifying DRG neurons in terms of size, CGRP production is known to be observed not only in small to medium neurons, but also in large DRG neurons. According to previous studies, the majority of small neurons have $\mathrm{C}$ fiber axons, which project to the superficial layers of the spinal dorsal horn. In contrast, the large neurons correspond to $A \alpha$ or $A \beta$ fibers, which project to the deep layers of the spinal dorsal horn or posterior nuclei. Previous studies demonstrated that there were shifts toward larger cell sizes in the size distribution of CGRP-IR DRG neurons and further CGRP expression in posterior nuclei increased in neuropathic pain models $[11,12]$. In addition, spontaneous activity and excitability of posterior nuclei in the nerve injury side were significantly higher than in the contralateral side [13]. Although these observations suggest that an up-regulation of posterior nuclei plays an important role in the generation and maintenance of pain, CGRP expression in posterior nuclei was not significantly affected by long-term immobilization in the present study. As evidenced by a retrograde tracing study, CGRP-IR fibers from medium-size lumbar DRG neurons project to posterior nuclei [16,25], but the projection of those from cervical DRG neurons is still unknown. Further research is needed to clarify which size cervical DRG neurons are the ones which predominantly project to posterior nuclei. 
In a previous report, endoneurial injection of nerve growth factor (NGF) increased the percentage of large-sized CGRP-IR DRG neurons [18]. Therefore, NGF seems to be implicated in a switch to CGRP expression in larger DRG neurons. However, the detailed mechanism of CGRP expression in the DRG, regulated by NGF,remains unknown. NGF also is induced by Schwann cells after nerve injury and is involved in the onset of pain behaviors in neuropathic pain [10,17]. In the current joint immobilization model, peripheral nerves might be injured by long-term casting. Having said that, there was no evidence of peripheral nerve injury or NGF up-regulation after joint immobilization.

In the deep layers, where CGRP expression was up-regulated by long-term immobilization, there was a distribution of wide-dynamic range (WDR) neurons. The WDR neurons have been proven to respond to both noxious and innocuous stimuli in electrophysiological studies. Growing evidence suggests that neuronal discharge of WDR neurons is involved in pathological pain conditions, such as allodynia and hyperalgesia. Allodynic rats showed a significant decrease in the proportion of low-threshold neurons and an increase in the proportion of WDR neurons [4]. Electrophysiological recording from WDR neurons in the spinal dorsal horn in hyperalgesic rats significantly increased spontaneous activity and after-discharges to noxious mechanical stimuli [29]. Furthermore, the administration of CGRP8-37, a selective CGRP receptor antagonist, induced a significant decrease in the evoked discharge frequency of WDR neurons [23]. Therefore, the up-regulation of CGRP expression in the spinal deep layers might modulate nociception through WDR neuronal activation in the present immobilization model. Indeed, we 
have previously demonstrated that the population of WDR neurons in the immobilized side increased after joint immobilization [27].

In conclusion, long-term casting induces the phenotypic changes in CGRP expression both at the DRG and spinal cord levels (specifically in deep layers) on the immobilized side. In terms of pathological pain states after long-term joint immobilization, these alterations may be of extreme importance. 


\section{References}

[1] S. Balasubramanyan, P.L. Stemkowski, M.J. Stebbing, P.A. Smith, Sciatic chronic constriction injury produces cell-type-specific changes in the electrophysiological properties of rat substantia gelatinosa neurons, J. Neurophysiol. 96 (2006) 579-590.

[2] S.H. Butler, M. Nyman, T. Gordth, Immobility in volunteers produces signs and symptoms of CRPS (1) and a neglect-like state, Abstracts: 9th World Congress on Pain. Seattle: IASP Press, 52 (1999).

[3] M.S. Cohen, H. Hastings, Post-traumatic contracture of the elbow. Operative release using a lateral collateral ligament sparing approach, J. Bone. Joint. Surg. Br. 80 (1998) 805-812.

[4] J.X. Hao, R.C. Kupers, X.J. Xu, Response characteristics of spinal cord dorsal horn neurons in chronic allodynic rats after spinal cord injury, J. Neurophysiol. 92 (2004) 1391-1399.

[5] F. Kaneko, T. Murakami, K. Onari, H. Kurumadani, K. Kawaguchi, Decreased cortical excitability during motor imagery after disuse of an upper limb in humans, Clin. Neurophysiol. 114 (2003) 2397-2403.

[6] I. Kangrga, M. Randic, Tachykinins and calcitonin gene-related peptide enhance release of endogenous glutamate and aspartate from the rat spinal dorsal horn slice, J. Neurosci. 10 (1990) 2026-2038. 
[7] S.G. Khasabov, D.T. Hamamoto, C. Harding-Rose, D.A. Simone, Tumor-evoked hyperalgesia and sensitization of nociceptive dorsal horn neurons in a murine model of cancer pain, Brain. Res. 1180 (2007) 7-19.

[8] K. Kuniyoshi, S. Ohtori, N. Ochiai, R. Murata, T. Matsudo, T. Yamada, S.S. Ochiai, H. Moriya, K. Takahashi, Characteristics of sensory DRG neurons innervating the wrist joint in rats, Eur. J. Pain. 11 (2007) 323-328.

[9] S.E. Lee, J.H. Kim, Involvement of substance P and calcitonin gene-related peptide in development and maintenance of neuropathic pain from spinal nerve injury model of rat, Neurosci. Res. 58 (2007) 245-249.

[10] L. Li, C.J. Xian, J.H. Zhong, X.F. Zhou, Lumbar 5 ventral root transection-induced up-regulation of nerve growth factor in sensory neurons and their target tissues: a mechanism in neuropathic pain, Mol.Cell.Neurosci. 23 (2003) 232-250.

[11] W. Ma, M.A. Bisby, Increase of calcitonin gene-related peptide immunoreactivity in the axonal fibers of the gracile nuclei of adult and aged rats after complete and partial sciatic nerve injuries, Exp. Neurol.152 (1998) 137-149. 
[12] K. Miki, T. Fukuoka, A. Tokunaga, K. Noguchi, Calcitonin gene-related peptide increase in the rat spinal dorsal horn and dorsal column nucleus following peripheral nerve injury: up-regulation in a subpopulation of primary afferent sensory neurons, Neuroscience. 82 (1998) 1243-1252.

[13] K. Miki, K. Iwata, Y. Tsuboi, R. Sumino, T. Fukuoka, T. Tachibana, A. Tokunaga, K. Noguchi, Responses of dorsal column nuclei neurons in rats with experimental mononeuropathy, Pain. 76 (1998) 407-415.

[14] S. Ohtori, K. Takahashi, T. Chiba, M. Yamagata, H. Sameda, H. Moriya, Phenotypic inflammation switch in rats shown by calcitonin gene-related peptide immunoreactive dorsal root ganglion neurons innervating the lumbar facet joints, Spine. 26 (2001) 1009-1013.

[15] R. Oku, M. Satoh, N. Fujii, A. Otaka, H. Yajima, H. Takagi, Calcitonin gene-related peptide promotes mechanical nociception by potentiating release of substance $\mathrm{P}$ from the spinal dorsal horn in rats, Brain. Res. 403 (1987) 350-354.

[16] J.K. Persson, B. Lindh, R. Elde, B. Robertson, C. Rivero-Melián, N.P. Eriksson, T. Hökfelt, H. Aldskogius, The expression of different cytochemical markers in normal and axotomised dorsal root ganglion cells projecting to the nucleus gracilis in the adult rat, Exp. Brain. Res. 105 (1995) 331-344. 
[17] M.S. Ramer, M.A. Bisby, Adrenergic innervation of rat sensory ganglia following proximal or distal painful sciatic neuropathy: distinct mechanisms revealed by anti-NGF treatment, Eur. J. Neurosci. 11 (1999) 837-846.

[18] G. Ruiz, J.E. Baños, The effect of endoneurial nerve growth factor on calcitonin gene-related peptide expression in primary sensory neurons, Brain. Res. 1042 (2005) 44-52.

[19] M. Shibata, K. Abe, A. Jimbo, T. Shimizu, M. Mihara, S. Sadahiro, H. Yoshikawa, T. Mashimo, Complex regional pain syndrome type I associated with amyotrophic lateral sclerosis. Clin. J. Pain. 19 (2003) 69-70.

[20] D.H. Smullin, S.R. Skilling, A.A. Larson, Interactions between substance P, calcitonin gene-related peptide, taurine and excitatory amino acids in the spinal cord, Pain. 42 (1990) 93-101.

[21] P.C. Staton, A.W. Wilson, C. Bountra, I.P. Chessell, N.C. Day, Changes in dorsal root ganglion CGRP expression in a chronic inflammatory model of the rat knee joint: Differential modulation by rofecoxib and paracetamol, Eur. j. Pain. 11 (2007) 283-289.

[22] H. Sun, K. Ren, C.M. Zhong, M.H. Ossipov, T.P. MalanJr, J. Lai, F. Porreca, Nerve injury-induced tactile allodynia is mediated via ascending spinal dorsal column projections, Pain. 90 (2001) 105-111. 
[23] R.Q. Sun, N.B. Lawand, Q. Lin, W.D. Willis, Role of calcitonin gene-related peptide in the sensitization of dorsal horn neurons to mechanical stimulation after intradermal injection of capsaicin.

J.Neurophysiol, 92 (2004) 320-326.

[24] R.Q. Sun, N.B. Lawand, W.D. Willis, The role of calcitonin gene-related peptide (CGRP) in the generation and maintenance of mechanical allodynia and hyperalgesia in rats after intradermal injection of capsaicin, Pain. 104 (2003) 201-208.

[25] M. Tamatani, E. Senba, M. Tohyama, Calcitonin gene-related peptide- and substance P-containing primary afferent fibers in the dorsal column of the rat, Brain. Res. 495 (1989) 122-130.

[26] A.J. Terkelsen, F.W. Bach, T.S. Jensen, Experimental forearm immobilization in humans induces cold and mechanical hyperalgesia, Anesthesiology. 109 (2008) 297-307.

[27] T. Ushida, W.D. Wills, Changes in dorsal horn neuronal responses in an experimental wrist contracture model, J. Orthop. Sci. 6 (2001) 46-52.

[28] K.S. Vikman, A.W. Duggan, P.J. Siddall, Increased ability to induce long-term potentiation of spinal dorsal horn neurones in monoarthritic rats, Brain. Res. 990 (2003) 51-57.

[29] H.R. Weng, J.V. Cordella, P.M. Dougherty, Changes in sensory processing in the spinal dorsal horn accompany vincristine-induced hyperalgesia and allodynia, Pain. 103 (2003) 131-138. 


\section{Figure legends}

Fig.1. Size distribution analysis of CGRP-IR in DRG neurons after joint immobilization. There were shifts toward larger cell sizes in the size distribution of CGRP-IR of immobilized (ipsilateral) side in DRG neurons (Kolmogorov-Smirnov test : $\mathrm{p}<0.0001)$.

Fig.2. Photomicrographs and histogram of CGRP-IR fibers in spinal dorsal horn $(\mathrm{A}, \mathrm{B})$ and cuneate nuclei (C,D) after joint immobilization. The results of histogram are expressed as means \pm SEM of pixel. CGRP-IR fibers in laminae I-II and cuneate nuclei were not significantly different between immobilized (ipsilateral) side and contralateral side. However CGRP-IR fibers significantly increased in laminae III-V of ipsilateral side (B, arrow) compared with contralateral side. ${ }^{*} \mathrm{p}<0.05$ 


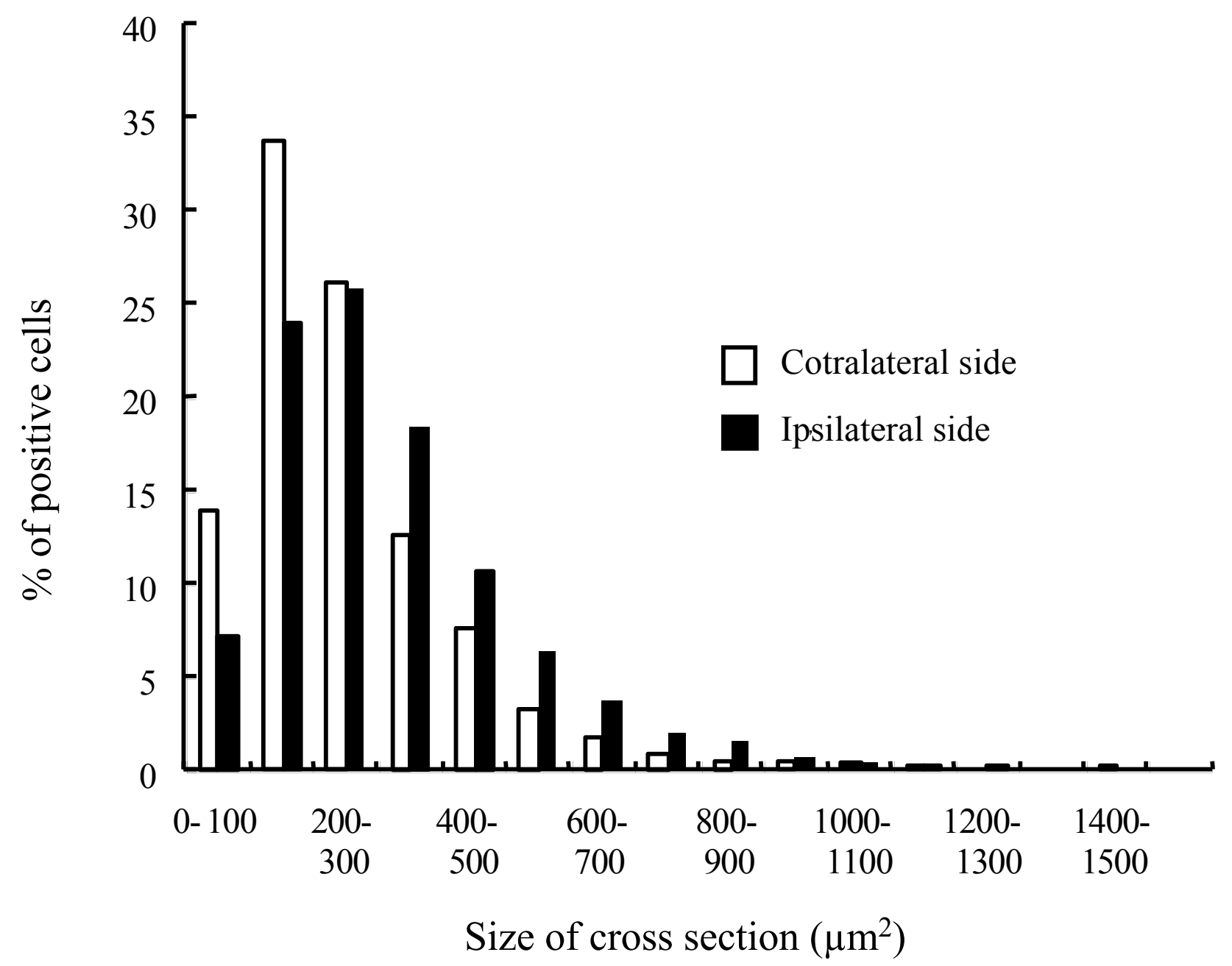

Fig. 1 
Contralateral side
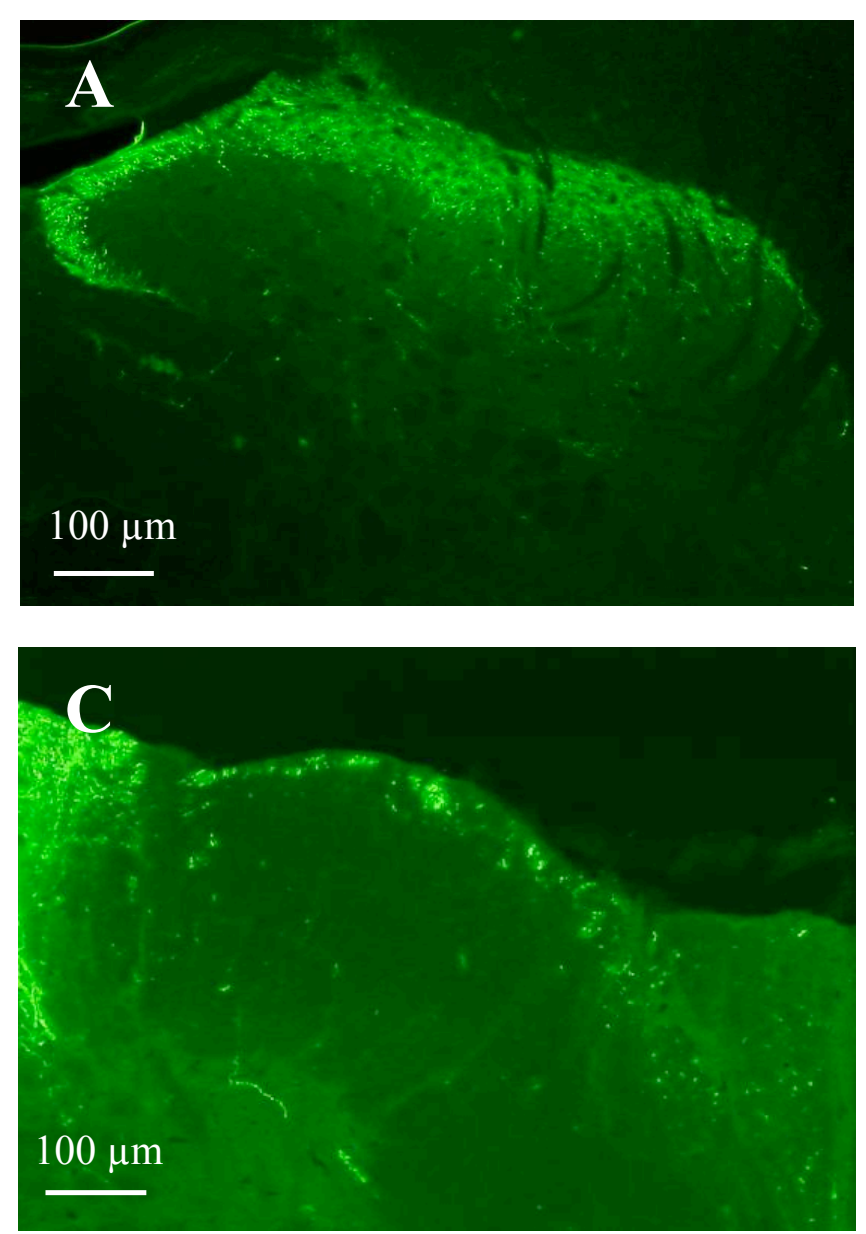

Ipsilateral side
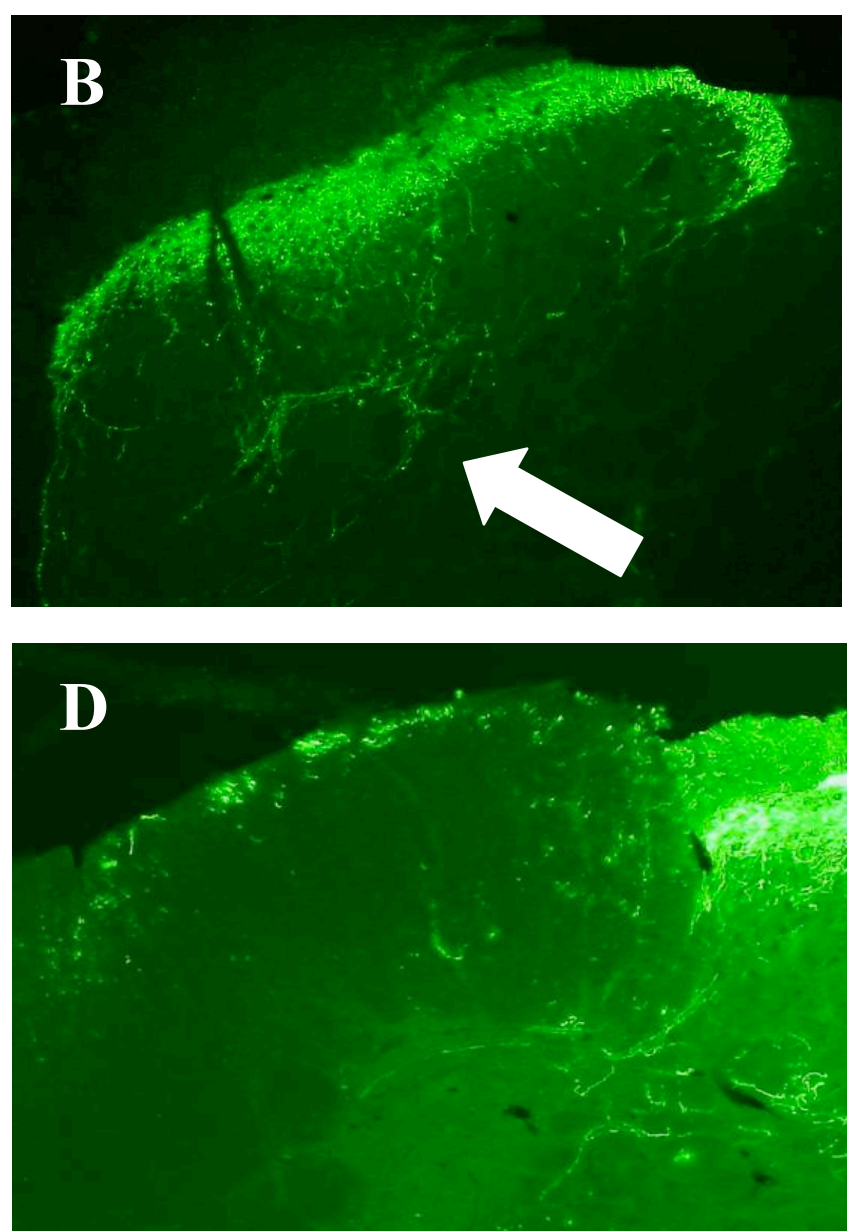
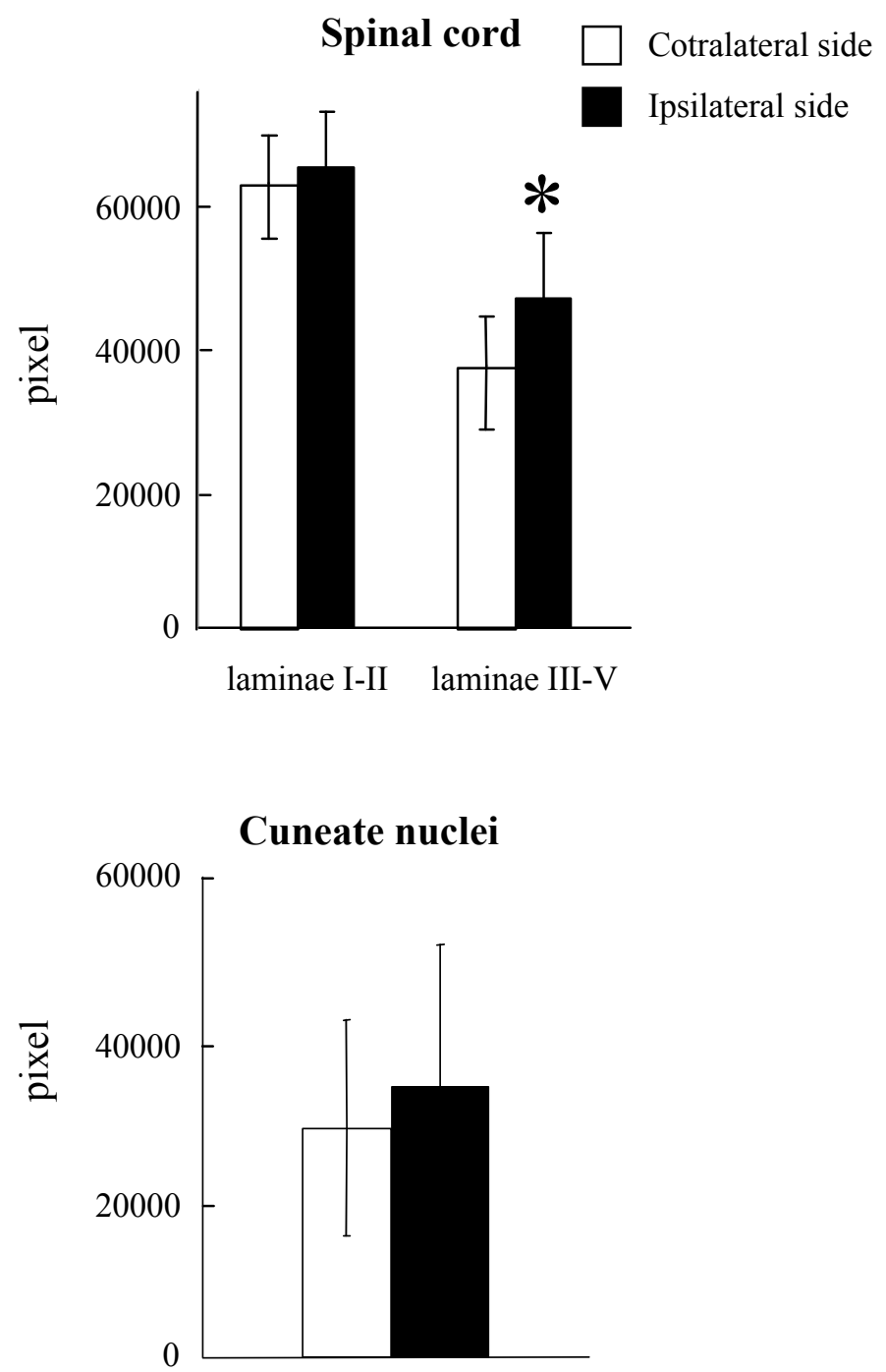